\title{
Justicia ambiental y políticas de reconocimiento en Buenos Aires
}

\author{
María Gabriela Merlinsky*
}

Perfiles Latinoamericanos, 26(51)

2018 | pp. $241-263$

DOI: $10.18504 / \mathrm{pl} 2651-010-2018$

\section{Resumen}

En las áreas más afectadas por la contaminación hídrica de Buenos Aires, se ha observado la construcción de demandas con las que diferentes organizaciones sociales cuestionan la desigualdad ambiental. En este artículo se analizan las características de estas movilizaciones a través de un estudio de caso, ponderando en su presentación pública y la construcción de los marcos de acción colectiva que definen la situación como un reclamo por justicia ambiental.

\begin{abstract}
In Buenos Aires, at the areas more affected by water pollution, we observe a process of construction of claims in which different groups question the environmental inequality. In this article we focus on a case study that examines the characteristics of these mobilizations, their public presentation and the construction of collective action frames in terms of environmental justice.
\end{abstract}

Palabras clave: justicia ambiental, acción colectiva, cuenca Matanza-Riachuelo, políticas de reconocimiento, cuestión hídrica, desigualdad ambiental.

Keywords: Environmental justice, collective action, Matanza-Riachuelo basin, political recognition, question water, inequality environmental.

* Doctora en Geografía por la Universidad París 8. Doctora en Ciencias Sociales por la Universidad de Buenos Aires. Investigadora en el Instituto de Investigaciones Gino Germani, Facultad de Ciencias Sociales, Universidad de Buenos Aires | merlinsk@retina.ar 


\section{Introducción}

E

los últimos años, en diferentes regiones de América Latina, los ciudadanos y organizaciones que reclaman por justicia ambiental han producido resistencias y formas de acción directa en contra de amenazas visibles en sus comunidades. Con ello han contribuido a mostrar que los peligros tóxicos y las localizaciones de actividades potencialmente peligrosas para la salud humana se superponen de forma implacable con la desigual distribución de los ingresos, la renta y la propiedad. Estas demandas no siempre se expresan mediante las consignas de las organizaciones ambientalistas y, muy frecuentemente, los mismos actores son reacios a considerarse a sí mismos como "ecologistas" (Guha, 1989).

Cuando los grupos sociales reclaman por las desigualdades debidas al impacto diferenciado de los problemas ambientales, movilizan diversos lenguajes de valoración sobre lo justo y lo injusto en determinadas elecciones institucionales. La justicia ambiental se refiere a la distribución de los beneficios y los daños —entre individuos, grupos sociales, regiones e incluso países—, y a las cargas - financieras y otras - ligadas a las políticas ambientales — prevención de riesgos, modelos de gestión, recomposición de ambientes dañados-. No se trata solo de estimar los efectos negativos de un ambiente degradado, sino también de definir cuáles serán los medios políticos y las regulaciones necesarias para remediar la situación, es decir, quién va a pagar por la recomposición y cómo se llevarán a cabo estas medidas.

En la metrópolis de Buenos Aires, en particular en las áreas más afectadas por la contaminación hídrica, se ha observado un proceso de construcción de demandas en el que con diferentes narrativas se cuestiona la desigualdad ambiental. La población reclama la protección de sitios no urbanizados, rechaza la implantación de megaproyectos inmobiliarios, pide el control de las actividades contaminantes, reivindica el derecho a acceder al agua y el saneamiento, reclama por políticas de suelo urbano para los sectores populares y exige medidas de asistencia sanitaria. La acción ciudadana para lanzar el alerta y reclamar la atención gubernamental se ha organizado en redes lábiles con denominaciones muy sugerentes: foros, asambleas ciudadanas ambientales, grupos autoconvocados y asociación intercuencas, entre otras (Merlinsky, 2014).

Este artículo retoma los resultados de una investigación longitudinal iniciada en 2005, la cual analiza las experiencias colectivas de organización para enfrentar la degradación ambiental en los territorios de la cuenca baja del río Matanza-Riachuelo (Merlinsky, 2013). El estudio retoma los barrios y asentamientos donde la falta de servicios esenciales, la degradación del suelo y la contaminación hídrica son un desafío cotidiano que altera las rutinas y mina las 
condiciones de supervivencia de las familias. En estos años se ha observado que en estos barrios se realiza un trabajo colectivo en pos de identificar las amenazas ambientales y establecer las causas de los problemas de salud imperantes. Esta actividad implica categorizar diferentes eventos y desarrollar diversas formas de acción colectiva. Son acciones que se han organizado en redes y agrupamientos que exigen el acceso a bienes, servicios y obras públicas y que aspiran a una justa reparación a los cuerpos afectados.

El objetivo es analizar las naturaleza de tales reclamos, su presentación pública y el modo en que contribuyen a elaborar los marcos de acción colectiva que definen la situación como un reclamo por justicia ambiental. Retomando aspectos centrales de la literatura sobre el tema, aquí se estudia en detalle el proceso simultáneo de creación de redes y construcción de marcos cognitivos para entender el problema. Se trata de elaborar un inventario de nuevos saberes y conocimientos para visibilizar las demandas, determinar los modos de atribución de responsabilidad, cómo se interpela a los poderes públicos e identificar alternativas de solución.

La estrategia teórico-metodológica ha consistido en abordar un estudio de caso instrumental (Stake, 1995). Para ello se ha seguido de cerca a una red de actores cuya base de acción se encuentra en el barrio Villa Lamadrid (municipio de Lomas de Zamora) y que recibe el nombre de Foro Hídrico. Para responder a las preguntas de investigación se ha utilizado el análisis de las narrativas (Abbott, 1992; Polkinghorne, 1995) de los actores y las acciones colectivas en torno a la degradación ambiental, tomando en consideración la construcción de marcos de injusticia (Gamson, 1988). La relevancia del caso elegido tiene que ver con la permanencia de los reclamos citados en el tiempo (es una experiencia que lleva ya dieciséis años, pero sus antecedentes se remontan hasta la década de 1980), razón por la que se han generado efectos y "bucles de retroalimentación” (Hilgartner \& Bosk, 1988) acerca del debate sobre la cuestión ambiental y social en la cuenca Matanza-Riachuelo.

Las fuentes de datos provienen de entrevistas en profundidad con pobladores de Villa Lamadrid y de entrevistas grupales e individuales con integrantes de Foro Hídrico. Se han recolectado además registros en talleres de capacitación y acompañamiento a las actividades de la organización, en el marco de las tareas de extensión universitaria de la Facultad de Ciencias Sociales de la Universidad de Buenos Aires (UBA). La primera serie de entrevistas en profundidad se realizó entre 2003 y 2004. Un segundo grupo de entrevistas se dio entre 2006 y 2007 (cuando formaron parte de una investigación-acción en la que se elaboró un diagnóstico comunitario en el barrio), y hubo un tercer relevamiento entre 2011 y 2014 como parte de un proyecto de investigación acerca de las políticas de recomposición ambiental de la cuenca Matanza-Riachuelo. 
Por otra parte, se ha recuperado material muy valioso de los registros de observación participante en distintas reuniones (audiencias públicas, solicitudes de información, reuniones con funcionarios) en las que se acompañó a los protagonistas de los reclamos.

Adicionalmente se recolectó información de prensa y transcripciones y registros fílmicos de reuniones y actividades de protesta. En paralelo se usaron registros del material fílmico Atravesando el río, el cual documenta el proceso de movilización social por el acceso al agua y el saneamiento de la cuenca Matanza-Riachuelo (Fernández Bouzo et al., 2006).

\section{Justicia ambiental y ocupación del suelo en la cuenca Matanza-Riachuelo}

El movimiento de justicia ambiental ha contribuido a introducir en la agenda política el nexo que existe entre el peligro ambiental y la desigualdad social. Su plataforma política ha consistido en exigir un tratamiento justo para todas las personas en lo que se refiere a la elaboración, desarrollo, implementación y aplicación de políticas, leyes y regulaciones ambientales. Por "tratamiento justo" se entiende que ningún grupo de personas, incluyendo los étnicos, raciales o de clase, deba soportar una cuota desproporcionada de las consecuencias ambientales resultantes de la operación de emprendimientos industriales, comerciales y municipales, de la ejecución de políticas y programas estatales, así como de las consecuencias por omisiones de estas políticas (Bullard, 1994; Acselrad, Campello \& Das Neves, 2009).

Para entender lo que está en juego en las luchas de los movimientos por la justicia ambiental es necesario prestar atención a los conflictos ecológicos distributivos por el acceso a los recursos que son esenciales para garantizar el modo de vida local (Fuks, 1996; Giuvant, 1998). La inscripción territorial de los reclamos coloca en el centro de la escena el problema del acceso a los recursos para la vida (agua, tierra, recursos forestales, acceso a la infraestructura básica) en el marco de disputas económicas, pero también en términos de valores culturales e identitarios (Harvey, 1996; Schlosberg, 2012; Acselrad, 2004; Vainer, 2004; Faber, 2005; Pellow \& Brulle, 2005; Firpo, 2012; Martínez, 2004).

Lo característico de estas manifestaciones es que se trata de respuestas en contra de intervenciones territoriales que han conducido al acaparamiento de los recursos (Blanchon, Moreau \& Veyret, 2009: p. 4). En tanto la definición del problema está mediada por significados sobre los efectos de la reproducción y la defensa de la vida, al calor del conflicto surgen conexiones entre humanos y no humanos y diferentes concepciones de lo común, entendidas como el ámbi- 
to y el horizonte para la construcción de configuraciones humano-ambientales socialmente más justas y más sólidas (Di Chiro, 1998: p. 112). En este sentido, el marco cognitivo que define la situación de injusticia da cuenta de la imposición desproporcional de los riesgos ambientales para las comunidades con menos recursos de información, financieros y políticos (Bullard, 1994; Acselrad, Campello \& Das Neves, 2009; Young, 1990).

Pero además de la injusta distribución de los peligros y la inequidad en el acceso a los recursos, los movimientos de justicia ambiental cuestionan el papel de las asimetrías culturales y valorativas respecto de cómo se define lo justo en determinadas decisiones institucionales (Waltzer, 1983; Fraser \& Honneth, 2006). Esto abarca la justicia distributiva — reparto de cargas, medidas de reparación-y la justicia procedimental, es decir, qué actores son considerados en un diferendo: si se reconocen sus intereses, si pueden participar y cuál es el balance de poder efectivo que producen esas acciones (Paavola, 2007: p. 96).

En la actualidad, en la cuenca Matanza-Riachuelo, diversas organizaciones territoriales que reclaman el cierre de basurales a cielo abierto, el control de la contaminación y la gestión de las inundaciones, exigen también medidas para acabar con la inequidad en materia de políticas públicas, las que por acción u omisión incrementan el riesgo ambiental. Al reclamar por las afectaciones ambientales y sus impactos en la salud plantean un problema de inequidad generacional en el proceso histórico de ocupación del suelo.

Recientemente se ha analizado la vulnerabilidad sanitaria de la población de la cuenca Matanza-Riachuelo (CMR) a partir de la incidencia combinada de riesgo sanitario, densidad habitacional y nivel socioeconómico. El resultado mostró que el $53 \%$ de ella se encuentra expuesta a elevados niveles de riesgo sanitario, esto es, 2004245 personas, de las cuales 541754 merecen especial atención por habitar en áreas de alta densidad demográfica (Aysa, 2009, Tomo I: p. 18). La población en situación de alta vulnerabilidad sanitaria tiene una cobertura de agua potable del $40 \%$ y una cobertura media de servicios de desagües cloacales de solo el 10\%. En las zonas de alta vulnerabilidad sanitaria, la pobreza se cuadriplica en relación con las áreas de vulnerabilidad sanitaria baja, alcanzando al $66 \%$ de los habitantes.

El área más crítica es la franja ribereña de la cuenca baja, donde cerca de trescientos mil individuos residen en villas, asentamientos y barrios populares, concentrados sobre todo en los municipios de Lomas de Zamora, Lanús, Avellaneda, La Matanza y en los límites con Riachuelo, en la Ciudad Autónoma de Buenos Aires (CABA).

Muchas de estas urbanizaciones ocupan suelos contaminados, con deficientes condiciones de habitabilidad y un déficit sanitario importante de agua y 
saneamiento cloacal. El rasgo fundamental es que se trata de áreas por debajo de la cota frecuentemente afectadas por inundaciones periódicas.

Considerando este déficit estructural en políticas de suelo urbano y sus efectos en términos de vulnerabilidad social y riesgo ambiental, es importante preguntarse por la capacidad de respuesta, es decir, de qué manera se definen los reclamos y el modo en que esto ha contribuido a modificar las narrativas en torno a la justicia ambiental.

\section{Ocupación del suelo y construcción de organizaciones territoriales en Lomas de Zamora}

El municipio Lomas de Zamora forma parte del primer cordón del conurbano bonaerense. En él viven 619279 habitantes, por lo que es el segundo partido más poblado del Gran Buenos Aires. En su interior se identifican dos áreas geográficas: las localidades ubicadas hacia el sudeste que se encuentran en zonas altas y las localidades ubicadas al noreste —aledañas al río MatanzaRiachuelo- que se emplazan en terrenos bajos e inundables.

\section{Mapa 1. Partido Lomas de Zamora: división interna y municipios limítrofes en la cuenca baja del Matanza-Riachuelo}

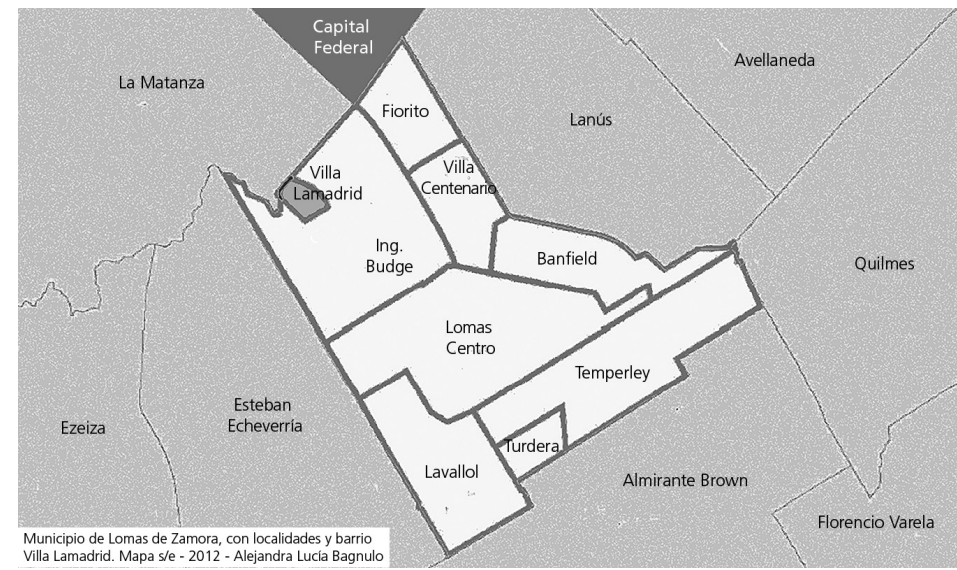

Fuente: Bagnuolo (2012)

En la metrópolis de Buenos Aires, la construcción de viviendas ha estado comandada por operaciones mercantiles destinadas a grupos de ingresos medios-altos y altos. Frente al bajo nivel de intervención estatal para dedicar 
suelo urbano a los sectores populares y en la construcción de vivienda social, la ocupación informal de tierra todavía es el principal medio de acceder al hábitat popular (Torres, 1993; Cravino, 2008).

Dada la ausencia de regulación del mercado de tierras, las áreas vacantes están sometidas a la especulación. Los pobladores de bajos recursos muchas veces pagan por un lote sin infraestructura y sin equipamiento social en los sitios inundables de las cuencas bajas. Al construir sus casas en zonas de peligro ambiental —bajo cota de inundación, sobre basurales y lugares contaminadosconsiguen una mejor comunicación con áreas centrales, las cuales facilitan hallar una plaza en el mercado de trabajo. Esta opción de autoproducción del hábitat popular significa un despliegue extraordinario de energía y fuerza de trabajo en pos de mejorar las viviendas, acceder al agua potable, rellenar terrenos y construir equipamientos. Por otra parte, como las más de las veces las ventas se dan sin los títulos de propiedad, estos pobladores carecen de seguridad en la tenencia de los terrenos.

En Villa Lamadrid las viviendas se fueron construyendo en las proximidades de los márgenes del Matanza-Riachuelo donde los terrenos son muy bajos y porosos. Debe decirse que, tratándose de un río de llanura, el curso de agua presenta bajo escurrimiento y se mueve por una leve pendiente ocupando los terrenos de modo irregular. En los testimonios de los vecinos con mayor tiempo en la zona, las referencias a las inundaciones periódicas se remontan a las décadas de los sesenta y los setenta, es decir, cuando comenzó el poblamiento del barrio. Sin embargo, lo que más se destaca en esos relatos es que la situación se fue agravando por el aumento de la densidad demográfica, por la falta de obras de infraestructura y por déficits en la regulación del uso del suelo.

A mediados de la década de los ochenta, diferentes barrios de la cuenca baja del Matanza-Riachuelo se organizaron para enfrentar las inundaciones periódicas, en particular por el desborde del Arroyo del Rey, uno de sus cauces tributarios. Así nació la Interbarrial, cuyo propósito principal era reclamar por obras de limpieza y mantenimiento de los arroyos, la realización de obras de defensa contra las inundaciones y la construcción de estaciones de bombeo (Foro Hídrico, 2003: entrevista colectiva por G. Merlinsky).

A comienzos de la década de los noventa se registró un nuevo crecimiento poblacional que surgía por la multiplicación de ocupaciones de tierras para la construcción de viviendas informales. Muchos de los pobladores recién llegados no tenían garantizada la cobertura de agua potable, por lo que este reclamo se unió al de los que ya vivían ahí y que, aunque habían logrado la conexión a la red, no contaban con conexiones cloacales. Entonces se creó la organización Agua para Todos, la cual asumió la tarea de reclamar a la empresa de agua y saneamiento a fin de que emprendiera obras para la provisión del servicio. Fue 
destacado el reclamo por la construcción de un "tercer refuerzo", esto es, un gran canal troncal que conectaría los barrios a la red de agua y saneamiento de la ciudad. En ese momento, los vecinos comenzaron a llevar registros de los habitantes sin cobertura y, por lo tanto, a reconocer que ello solo podía solucionarse mediante inversiones estatales en obras públicas de gran envergadura. Fue un periodo en el que se reconoció el carácter estructural del problema (V. L., vecino de Lamadrid, 2006: entrevista por G. Merlinsky).

Por esta época, buena parte de los reclamos iban dirigidos a Aguas Argentinas, la concesionaria prestadora del servicio a partir de su privatización, en 1993. En los primeros años de gestión, dicha empresa se propuso llevar el líquido desde el área central de la ciudad a los municipios de la cuenca baja mediante un conducto que los aprovisionaría extrayendo agua de la fuente superficial del Río de La Plata. Como el criterio dominante para la expansión de las obras fue garantizar la rentabilidad del servicio, no se incluyeron las obras de conexión cloacal. La construcción del "tercer refuerzo" se llevó a cabo con cierta celeridad y, una vez concluida, la empresa decidió cerrar los antiguos pozos que había en la zona.

En 1997 aparecieron las primeras irregularidades: dado que estos cambios no se acompañaron de una inversión equivalente para expandir la red cloacal, se contribuyó a acentuar el desbalance hídrico y, por ser un territorio bajo cota de inundación, las napas freáticas comenzaron a aumentar su nivel. En 2000 se produjo una gran inundación en la zona durante la temporada de lluvias, y el agua tardó en retirarse por la dificultad de absorción de los suelos. En esa ocasión hubo veinte mil evacuados y se declaró la emergencia hídrica en el municipio, fue entonces que los vecinos fundaron el agrupamiento Foro Hídrico. Esta inundación extraordinaria representó un punto de inflexión para la agregación de demandas colectivas y la redefinición tanto de la escala del problema como de la asignación de responsabilidades institucionales. Si bien el núcleo de la organización surgió de Villa Lamadrid, se incorporaron vecinos y grupos de Lomas de Zamora y de Almirante Brown, Lanús, Avellaneda y La Matanza.

Los mismos vecinos y organizaciones territoriales que habían sido parte de la lucha por "el tercer refuerzo" impulsaron la convocatoria para el nuevo agrupamiento, con el fin de enlazar demandas que antes habían corrido por vías de acción separada: la provisión del agua, el saneamiento cloacal, el control de las inundaciones y los problemas de salud.

Como señalaba Juan Walter, uno de los líderes barriales más reconocidos en la zona,

había muchísima participación, llegamos a ser doscientos en la asamblea y había más de cincuenta instituciones participando, fue así que hubo luchas muy grandes 
en torno a esta temática; nosotros ampliábamos la petición, no nos centrábamos en el problema de las napas, decíamos que la solución era mucho más amplia, fuimos dándonos cuenta que para solucionar el problema hídrico en la zona se necesita tener un proyecto integral (Juan Walter, integrante de Foro Hídrico, 2004: entrevista por G. Merlinsky).

En esta otra etapa - y ante las importantes controversias con la propia empresa en relación con las causas de estas inundaciones extraordinarias-, los vecinos recurrieron al Instituto Nacional del Agua (INA), por un lado, y a la Facultad de Ingeniería de la UBA, por el otro, para solicitar información respecto de las causas del problema. Al mismo tiempo y dado que en el área se registraba un aumento de las enfermedades hídricas, se empezaron a organizar reuniones con los médicos de la zona, para coordinar la implementación de un diagnóstico de salud siguiendo la línea de indagación de la epidemiología comunitaria (Merlinsky, 2013).

Todo lo anterior dio lugar a espacios de experimentación y a la elaboración de inventarios de problemas y soluciones. Y esto contribuyó a cuestionar las definiciones basadas exclusivamente en el conocimiento de los "expertos" y a confrontar las decisiones de las burocracias estatales y los círculos empresariales. Hasta la fecha, el poder de movilización de Foro Hídrico reside en su formato lábil, en la heterogeneidad de los miembros involucrados — expertos, vecinos, profesionales de la salud, profesores universitarios, representantes de organizaciones territoriales, líderes políticos, etc.- - y en la apelación a diversos registros —éticos, económicos, sociales, culturales, de construcción de derechos - para la definición de los problemas comunitarios (Callon et al., 2001: p. 36). La producción de conocimiento local y la construcción de redes "contraexpertas" son el cemento que mantiene unida la red y le da visibilidad en la esfera pública.

\section{Foro Hídrico como red "contraexperta". ¿Qué conocimiento cuenta y quién lo produce?}

Si bien Foro Hídrico es una organización compuesta por vecinos de diversas localidades sigue un formato de acción colectiva marcadamente territorial: los barrios son el lugar de residencia y desde ellos se define y sostiene la lucha. Por esta razón, los vecinos se refieren a las organizaciones territoriales que integran Foro Hídrico conectando la experiencia actual con la historia de organización previa. Durante más de veinte años, diferentes integrantes del actual Foro Hídrico habían participado en sociedades de fomento, comités de inundados, 
grupos de articulación por cuenca, asambleas ciudadanas y organizaciones vecinales que reclamaban y aún exigen la construcción de escuelas, la realización de infraestructuras, el acceso al agua potable, el tendido de caminos y la gestión del riesgo de inundaciones, entre otras. Como parte de estas redes, en muchas ocasiones habían realizado múltiples e ingeniosas formas de protesta: acampes, cortes de ruta, encadenamientos en Plaza de Mayo, festivales para hacer público el reclamo, producción de videos colectivos, campañas epidemiológicas, etcétera.

Cuando consultamos a los participantes sobre el origen de Foro Hídrico, la respuesta aludía a "lanzar el alerta" (Bernstein \& Jasper, 1998), lo cual es propio de los movimientos que reclaman por afectaciones ambientales. Se trata de la apertura de una temporalidad que se distingue por los procesos de asociación, cooperación, comunicación y movilización pública. Como consecuencia, se extienden los marcos de injusticia hacia un conjunto organizacional más amplio (Cefaï, 2007: p. 576), se genera cierta influencia en la opinión pública y se producen reacomodamientos políticos en torno a los modos de acción colectiva (Snow et al., 1986). Señalaba Alfredo, uno de los activos participantes de Foro Hídrico:

la organización se hizo mucho más fuerte, pero además amplió la visión que fuimos teniendo del problema y como se rechazaban las causas que nosotros planteábamos (fundamentalmente de parte de Aguas Argentinas), como ellos no se hacían cargo, eso nos llevaba a buscar las razones. Y sobre todo, buscar aliados, porque estamos hablando del tránsito del gobierno de Menem a la Alianza, entonces muchos políticos participaban por esa razón. Y llegó en aquel entonces en el año 2000, la gran inundación que fue un golpe fuertísimo, es allí cuando decidimos, en julio del 2000, tener una herramienta que se llamaría Foro Hídrico. Además tomábamos nuestros recaudos con los partidos políticos para lograr apoyos de diferentes agrupaciones. Nosotros ampliábamos la petición, no nos centrábamos en el problema de las napas, decíamos que la solución era mucho más amplia. Fuimos dándonos cuenta que para solucionar el problema hídrico en la zona se necesita tener un proyecto integral; no bastaba con las bombas depresoras que era nada más que paliativos [...] (Alfredo, Foro Hídrico, 2006: entrevista por G. Merlinsky).

En ese momento de mayor activación de la demanda, vecinos de diferentes barrios se reunieron en asambleas en donde se debatía, compartía conocimientos y se monitoreaban las formas y tiempos de protesta. Son estas deliberaciones las que, al decir de Callon et al. (2001), extienden la discusión sobre "estados de mundo posibles", con la intervención de una pluralidad de puntos de vista 
y eventuales respuestas. Se trata de dispositivos de exploración y de aprendizaje colectivo en los que se analizan los problemas, se buscan pruebas y se discute quiénes son los actores acreditados para generar conocimiento válido. Esto condujo a un cuestionamiento de las acciones del Estado y de las corporaciones en términos de una economía política del conocimiento (Sismondo, 2010). Esto es, se generaron debates sobre la legitimidad de los saberes, aspecto que se resume en dos preguntas importantes: ¿cuál es el conocimiento que cuenta? y ¿quién lo produce?

Durante los últimos años la autora de este artículo ha acompañado a Foro Hídrico asistiendo a reuniones, a actividades de protesta, a debates públicos y a asambleas. Con la intención de elaborar un inventario de los argumentos de esta organización se ha mejorado nuestra comprensión acerca de los modos colectivos de definición de los eventos, la búsqueda de información y la identificación de las causas de los problemas.

Cuando se ha consultado a los integrantes por los objetivos de la organización, se menciona reiteradamente el derecho a la información confiable y a tener injerencia en las decisiones públicas sobre las obras, equipamientos e infraestructuras que son necesarias para superar la situación de injusticia. Como la ejecución de las obras de infraestructura se encuentra atada a decisiones importantes respecto de las opciones tecnológicas, plazos y modos de implementación, los vecinos han aprendido que los logros dependen de un seguimiento muy directo en el control de gestión. A partir de allí, la traducción del reclamo al lenguaje de la justicia ambiental se construye con base en tres cuestiones relacionadas con el derecho a la información, el derecho humano al agua y con la participación en las decisiones de política pública que tienen consecuencias en la vida comunitaria

\section{El derecho a la información y la construcción de conocimientos alternativos}

En la literatura sobre los movimientos de justicia ambiental se ha afirmado que uno de sus factores detonantes es que las agencias estatales niegan el problema, aspecto que muchas veces incluye el ocultamiento sistemático de la información. Esta falta de reconocimiento simbólico de las víctimas y su destitución en tanto actores con derecho a saber es, en no pocas oportunidades, lo que impulsa el reclamo de justicia. Las víctimas cuestionan la retórica técnico-burocrática y le contraponen un lenguaje para humanizar las nociones que definen y nombran el problema (Capek, 1993; Di Chiro, 1998; Gibbs, 1992); es entonces que la percepción de ser tratado como "ciudadano/a de segunda 
categoría" funciona a modo de poderoso activador de la movilización social (Capek \& Gilderbloom, 1992).

Por otra parte, esa falta de información induce a los actores a desarrollar formas propias de construcción de conocimiento. Se trata de experiencias de epidemiología popular (Brown, 1992) en las que los actores locales investigan por su cuenta para determinar las causas de las enfermedades. Buscando datos e información en fuentes diversas, los ciudadanos procuran identificar cadenas causales de mayor alcance - no solo los nexos sociales y demográficos de la epidemiología tradicional- para determinar los niveles de responsabilidad en la generación del daño. De este modo, la búsqueda de pruebas incluye los intereses empresarios, las decisiones gubernamentales y las normas de regulación (Akrich, Barthe \& Rémy, 2010: p. 19).

La construcción de herramientas de prueba alternativas comenzó a mediados de la década de los noventa, cuando los vecinos de Foro Hídrico vivían las inundaciones por el elevamiento de las napas freáticas y Aguas Argentinas negaba que su solución técnica tuviera relación con el problema. Para confrontar esta respuesta, Foro Hídrico recurrió a otras fuentes de conocimiento experto, en concreto, al Instituto Nacional del Agua (INA), el cual realizó un estudio independiente. La conclusión fue que las incorrectas decisiones técnicas de Aguas Argentinas habían llevado un peso decisivo en las inundaciones (INA, 2003).

Cuando se consultó a un integrante de Foro Hídrico, él señalaba que esta búsqueda de información ha sido parte de la lucha y de ganar un espacio en la negociación con los organismos estatales. En sus palabras: "nosotros siempre fuimos buscando datos y presentando proyectos alternativos, cuando se publicó el estudio del INA, lo bueno es que era una confirmación de lo que nosotros pedíamos, nos dio como una cierta autoridad moral" (Víctor F., integrante de Foro Hídrico, 2006: entrevista con G. Merlinsky).

En años recientes el derecho a la información forma parte de los reclamos colectivos por afecciones a la salud y los relevamientos comunitarios tienen el fin de evidenciar una situación de "emergencia sanitaria". Para ello han realizado distintos estudios acerca de la situación de salud en los barrios junto con la organización Médicos del Mundo y la Cátedra Libre de Ingeniería Comunitaria de la Facultad de Ingeniería de la UBA. El objetivo ha sido producir datos que demuestren los nexos entre los problemas de contaminación, la falta de servicios sanitarios y la situación de salud a escala local. Es clave que no hay estadísticas que muestren dichas correlaciones, porque hospitales y centros de salud de la región no aplican estudios epidemiológicos y carecen de registros de historias clínicas que tomen en cuenta la relación entre lugar de residencia, peligros ambientales y afecciones a la salud. 
En 2011, Foro Hídrico, al lado de Médicos del Mundo, inició la campaña "Paremos con el genocidio silencioso de nuestro pueblo", que consistía en el diseño y la implementación de encuestas epidemiológicas en Villa Lamadrid. Los resultados mostraron que $62 \%$ de las familias sufre inundaciones en su casa o terreno, $100 \%$ no cuenta con servicio de cloacas, $34 \%$ consume agua envasada, el barrio no cuenta con servicio de recolección de residuos y $75 \%$ de la población no tiene cobertura médica. Asimismo, el estudio determinó la existencia de enfermedades respiratorias, parasitosis, gastroenteritis y dermatitis (Argenpress, 2014).

Luego de la experiencia en Villa Lamadrid, y en el marco de la misma campaña, se replicó la encuesta entre cien familias del barrio 17 de Noviembre, un asentamiento localizado sobre rellenos industriales y atravesado por efluentes contaminados. En este relevamiento participaron una veintena de organizaciones, agrupaciones políticas, movimientos sociales y grupos universitarios, como la Cátedra Libre de Ingeniería Comunitaria y la Cátedra Libre de Estudios Americanistas, ambas de la UBA. Entonces se concretaron actividades de información en la vía pública con las que se difundieron los resultados del estudio y se generaron puntos de recepción de consultas y denuncias. Esto convocó la atención de la prensa y las voces de los vecinos aparecieron en las coberturas periodísticas. Un residente del barrio 17 de Noviembre señalaba: "yo estoy desde el comienzo acá. Todos vimos cuando traían, con los camiones de la municipalidad, volquetes con basura, residuos patológicos, barro del Riachuelo. Dejaban de todo, total, nadie controlaba nada". Irma, otra vecina, comentaba: "cuando está nublado o hay mucha humedad, el olor a podrido no se aguanta". Amalia, la mamá de Lucas, un niño de un año, expresaba: "mi hijo tiene una colostomía, que le hicieron al nacer. Me dijeron que podía deberse a cuando nuestra casa estaba rodeada de agua podrida, que eso podía ser la causa, pero no sé yo" (Argenpress, 2014).

La producción y difusión del conocimiento permite que una diversidad de actores se articule para incidir en la opinión pública y/o visibilidad por su rol tecno-científico. La información producida localmente es fundamental para mostrar el problema, fundamentar la legitimidad del reclamo, lograr influencia en la opinión pública y dejar en claro que existe un derecho violentado.

\section{El derecho humano al agua}

En 2002, el Comité de Derechos Económicos, Sociales y Culturales, organismo de las Naciones Unidas destinado a supervisar el cumplimiento del Pacto Internacional de Derechos Económicos, Sociales y Culturales (PIDESC), reconoció en su observación núm. 15 que el agua es un derecho humano universal, 
inalienable e inviolable. Asimismo, allí queda consignado que el acceso al agua se vincula al derecho a la salud (artículo 12) y al derecho a un nivel de vida adecuado (artículo 11). Así, el acceso al agua y a las cloacas son derechos universales, lo que implica hacerlos accesibles a toda la población con independencia de sus condiciones económicas particulares, es decir, que se deben ofrecer solo por ser ciudadanos (Tobías, 2016).

En los últimos años, las narrativas y repertorios de acción de los grupos y activistas de Foro Hídrico se concentran en desarrollar campañas para hacer exigible tal derecho y lograr que se concrete en acciones efectivas y afirmativas de las políticas públicas.

Desde la perspectiva de estos actores, en Buenos Aires ha sido la discontinuidad de las obras públicas la que, al compás de las oleadas de privatización y reestatización, ha dejado como saldo una brecha histórica de inequidad en la cobertura de servicios. En efecto, si en la Capital Federal el servicio cubre al total de la población, en la zona norte del área de concesión de Agua y Saneamientos Argentinos S. A. (AysA), en la Región Metropolitana de Buenos Aires, ese porcentaje se reduce al $72 \%$. Mientras que, en la zona sur que es el área que coincide parcialmente con la cuenca Matanza-Riachuelo, solo el 59\% de la población cuenta con servicios sanitarios (AysA, 2013).

Cabe destacar que la fórmula del desarrollo sustentable, consistente en que el mismo no ponga en riesgo los derechos de las generaciones futuras, en la versión de estos actores movilizados hay una deuda histórica marcada por la oportunidad de las clases medias y altas para apropiarse de los recursos hídricos en desmedro de los sectores populares.

En 2006, el servicio de agua fue reestatizado y pasó a manos de AysA, una sociedad del Estado. El diagnóstico reportaba entonces un déficit de cobertura de tres millones de personas sin servicio de agua potable y seis millones que carecía de desagües cloacales. Fue en ese momento que la empresa diseñó un Plan Director con la meta de llegar al cien por ciento de cobertura para 2020. En los últimos diez años (2006-2016) hubo avances significativos en el tendido de la red de agua, pero la evolución fue mucho menor respecto de la red cloacal, entre otras cosas porque ello depende de obras de mayor envergadura que permitan ampliar la capacidad del sistema mediante colectores como condición previa para construir redes que soporten las nuevas obras.

En los últimos años, las organizaciones de Villa Lamadrid han activado campañas para mostrar que esa brecha histórica se explica porque ha primado el criterio de rentabilidad en la lógica de provisión del servicio, precisamente porque es más rápido y seguro recobrar la inversión cuando se trata de provisión de agua potable y, en contraste, se requieren grandes erogaciones para garantizar las obras del tamaño necesario para expandir la red cloacal. 
A partir de 2012, Foro Hídrico, el Departamento de Hidráulica de la Facultad de Ingeniería de la uba y la Fundación Che Pibe, encabezaron la campaña "El agua no es confort, el agua es vida", aludiendo a la necesidad de garantizar el acceso al agua y a las cloacas como derecho esencial y buscando mostrar las diferentes caras de la desigualdad territorial en el acceso a los servicios básicos: mientras algunos vecinos poseen la red de agua de AysA, otros se abastecen mediante redes vecinales, perforaciones (sin control de calidad) e incluso por medio de camiones cisterna o conexiones clandestinas (Informe Foro Hídrico. Causa Riachuelo, 2015).

La falta de cloacas en las zonas de mayor riesgo sanitario no es una casualidad. No es que la gente no tenga cloacas porque no tiene ganas, sino que siempre son los pobres los que han sido relegados por el negocio inmobiliario, por las empresas. Por ejemplo, cuando el servicio de osn fue privatizado se priorizó dar servicio a gente que pueda asegurar el pago de la tarifa, y no a gente que no pueda pagarlo. [...] Sin embargo, a la larga, los costos de no hacer las obras públicas las está pagando el Estado a través de la salud. (A. A., integrante de Foro Hídrico, 2013: entrevista por G. Merlinsky).

Para los integrantes de Foro Hídrico — que han recibido con beneplácito la reestatización del servicio-, esta demora en la implementación del Plan Director constata que hay barreras políticas en la definición de prioridades de obras. Las organizaciones cuestionan el modelo de racionalidad técnica que requiere de inversiones de largo plazo y la realización de grandes obras de infraestructura para dar paso a los colectores cloacales (construcción de uno nuevo en el margen izquierdo de Riachuelo) como prerrequisito para ampliar este tipo de cobertura. Todo ello conduciría a que la provisión del servicio demoraría entre quince y veinte años. El argumento de Foro Hídrico cuestiona este modelo de análisis de costo-beneficio, enfatizando en que la satisfacción de las necesidades de la población que está en riesgo sanitario no puede ser postergada. En estos argumentos, el problema se inscribe públicamente como un reclamo al Estado por la discontinuidad en las obras de infraestructura y por la falta de criterios de justicia ambiental que puedan compensar las inequidades en el acceso a los recursos.

\section{Participación en las decisiones de política pública con efectos en la vida comunitaria}

En la experiencia de organización que aquí describimos se constata otro elemento propio de los movimientos de justicia ambiental que se relaciona con 
el reclamo hacia el Estado y las políticas públicas en cuanto a acciones u omisiones estructurantes del problema. Así, los repertorios de reclamos aspiran a influir en las decisiones sobre los modos de implementación de las soluciones propuestas.

Este diagnóstico deriva de evaluar la experiencia histórica reciente. Conseguir el "tercer refuerzo" consumió una década de luchas, movilizaciones, reclamos y propuestas. La conquista de soluciones parciales para la construcción de canales de desfogue para paliar los efectos de las inundaciones también provino de la acción vecinal presentando proyectos y discutiendo alternativas de obra. El aprendizaje es que los reclamos deben sostenerse en el tiempo con propuestas concretas de intervención y, dado que se trata de infraestructura, se requiere además de la discusión de las opciones técnicas de implementación, plazos y modalidades de intervención. En este sentido, la movilización para la búsqueda de soluciones que incidan en la implementación de la política pública es un elemento central. Un entrevistado señalaba,

Cada vez que surge un problema y que lo identificamos como un problema común, buscamos soluciones comunes y luego nos movilizamos en búsqueda de ese tipo de solución. Nosotros nunca vamos a una protesta, sin una propuesta superadora de lo que el gobierno propone. Es decir, siempre llevamos una solución, no es que decimos "queremos cloacas y listo", les decimos que las queremos de esta manera y en tales plazos, con tales parámetros. O sea sabemos lo que queremos, y tratamos de divulgarlo en el barrio, a través de asambleas, o de ir a una escuela y hablar con los chicos, con los papás. Es un trabajo de formación, de formar conciencia, de llamar a la movilización y después nos reunimos y tratamos, con fuerza, de imponer las ideas. (A. L., integrante de Foro Hídrico, 2014: entrevista por G. Merlinsky).

A partir de un análisis del Plan Director de aysa, los vecinos de Foro Hídrico han concluido que es un modelo centralizado que requiere de construir grandes colectores y emisores (incluidos y luego demorados o suspendidos en los planes de anteriores gobiernos), lo que supondría demorar la terminación de las obras en una o dos décadas. En contrapartida, alientan la construcción de plantas modulares que brindarían cobertura en corto tiempo.

[En referencia a las grandes obras del Plan Director] Es un proyecto muy a largo plazo. Y va a depender no solo de la acción política de este gobierno, sino de los gobiernos futuros. Estamos hablando de que acá a quince años esas obras terminen. Yo no sé si realmente lo que dicen en quince años se termina, viene otro gobierno y dice: "No, espera, hay un proyecto mejor, vamos a hacer una planta espacial. Y cambian el proyecto y lo ponen al ańo 2045. Entonces lo que nosotros 
decimos es que hagamos las cloacas ya, después si teniendo cloacas se les ocurrió otra cosa, y bueno, hagan lo que les parezca. (A. A., integrante de Foro Hídrico, 2013: entrevista por G. Merlinsky).

Es una forma de territorializar la demanda, logrando que las obras lleguen rápido sin supeditar el proceso a los plazos de licitación y las pujas sectoriales por la distribución del financiamiento de las obras.

En este hecho se identifica un reclamo por justicia distributiva (que las obras lleguen al territorio involucra la compensación de un déficit histórico) y un reclamo por justicia procedimental. Esta última significa tomar en cuenta los mecanismos de participación, observando si se produce un cambio efectivo en el balance de las relaciones de poder en favor de los afectados.

En la experiencia de Foro Hídrico, acceder a la toma de decisiones sobre las obras siempre ha dependido de la acción directa y de presionar ante los organismos jurisdiccionales competentes. Por ello, una cuestión central es que la obra pública cada cierto periodo de tiempo entra en un impasse, lo que lleva a "ganar la calle" otra vez para incidir en el avance de la implementación.

¿Es la planta de tratamiento de efluentes cloacales de Dock Sud la solución? El tiempo lo dirá. Es una megaplanta para miles de personas que está planificada, fundamentalmente, para Capital y zona norte que van los efluentes [...] El hecho es, cortamos ahí, no dejamos llegar hasta ahí, entonces toda la capacidad de lo que venía se lo damos a la zona sur. Entonces, nosotros tenemos que continuar movilizados, porque si no, no vamos a conseguir las obras. (A. A., integrante de Foro Hídrico, 2013: entrevista por G. Merlinsky).

En tanto la provisión de agua y saneamiento es consecuencia de inversiones constantes en el tiempo, un retraso en los planes de ejecución de obras aumenta las brechas en el acceso a tales servicios. Y ello se agrava porque el crecimiento poblacional es más alto donde son mayores los déficits de inversión. Los habitantes de la cuenca baja del Matanza-Riachuelo han aprendido que las medidas de largo plazo requieren de horizontes políticos y económicos estables y sostenidos.

Los voceros de la empresa de agua y saneamiento consultados informaron que la prioridad era consolidar un plan de obras elaborado hace varias décadas. Sin embargo, un argumento dominante para no avanzar sostiene que se trata de suelos "no aptos" para grandes obras de infraestructura. La emergencia de organizaciones como Foro Hídrico ha permitido abrir esa discusión y, sobre todo, replantear las obras buscando alternativas tecnológicas que provean de los servicios en el corto plazo. 


\section{Reflexiones finales}

El objetivo de este artículo ha sido analizar el proceso de elaboración de reclamos por afectaciones ambientales de movimientos populares que exigen las mismas oportunidades de las clases altas y medias en cuanto al acceso a los beneficios de la centralidad urbana. Este proceso genera modos de participación y concepciones de ciudadanía que se reformulan desde las propias bases del derecho a la inclusión social (Holston, 2009: p. 46). Mediante un estudio de caso se ha mostrado el proceso simultáneo de creación de redes y construcción de marcos cognitivos para entender la injusticia ambiental.

En Buenos Aires se observa un proceso de construcción de ciudadanía por medio de demandas territoriales por agua, saneamiento y cierre de sitios contaminados, lo que plantea un reclamo fundante por el derecho colectivo, social y comunitario a la salud. En el caso analizado destaca la construcción de una red de organizaciones y acciones colectivas que abren campos de autonomía en la esfera social y cultural. Este es un rasgo propio de dichos movimientos, lo cual es un signo de época en relación con los movimientos sociales que, en la Argentina de los noventa habían surgido como respuesta a las políticas neoliberales. En efecto, buena parte de aquellas resistencias, organizadas a nivel barrial, buscaron la solución colectiva de necesidades más urgentes. En la construcción de reclamos por medios de subsistencia, estos movimientos asumieron una dinámica dual de confrontación-colaboración, pues solo así podían garantizarse los recursos para sostener acciones diarias como espacios educativos, comedores, viviendas, etc. Una característica distinta de estos movimientos para el nuevo milenio es que su funcionamiento no depende de recursos provistos por el Estado. Por el contrario, la interlocución con este en esta nueva fase es más confrontativa: los ciudadanos contemporáneos exigen mayor protección o regulación de los bienes ambientales y denuncian la inacción estatal en materia de política pública. Asimismo, la acción de estos colectivos se plantea como un espacio alterno a los partidos políticos.

La composición de estos grupos es multisectorial, reclutan a profesionales y expertos tanto de la comunidad como de organizaciones externas en defensa del ambiente. Así, surge una alianza de actores heterogéneos, de alta productividad en la construcción de saberes locales sobre el territorio y el ambiente. La gran complejidad y multidimensionalidad de la cuestión ambiental es en este caso un factor condicionante. En cada debate que se multiplica es necesario definir asuntos tan diversos y complejos como el nivel de peligrosidad de determinadas sustancias, la escala de producción y efluentes tolerables por el ecosistema de un río, el impacto admisible para la salud del uso de determinados compuestos o la forma adecuada de extraer agua para consumo humano sin dañar las napas, 
etc. Participar en estas discusiones, en consecuencia, requiere del aprendizaje de un saber científico y frecuentemente son los profesionales de cada comunidad o los docentes e investigadores universitarios que llevan adelante experiencias de acompańamiento comunitario.

Pero no solo se trata de la construcción de saberes contraexpertos. El saldo más interesante es que se generan nuevas experticias sobre el territorio. Los pobladores hacen valer su conocimiento y se autoperciben con autoridad para reclamar y oponerse a las amenazas contra su salud. Al cuestionar la de los expertos, ellos generan alertas sobre peligros y sustancias potencialmente dañinas. De este modo, se asumen como portadores de un conocimiento válido que abre interrogantes sobre la calidad de vida, la salud y el bienestar de la comunidad.

La clave en la construcción de los marcos que definen la justicia ambiental se relaciona con la exigencia de reconocimiento. Se trata de un aspecto central en la justicia procedimental que implica tomar en cuenta la visibilidad de las organizaciones sociales a través de sus prácticas representativas, interpretativas y comunicativas de la propia cultura (Fraser \& Honneth, 2006). El "no reconocimiento" es un tipo de opresión que deforma y moldea la concepción que dichos seres humanos tienen de sí (Taylor, 1993). Es por eso que el movimiento de justicia ambiental exige un igual trato ante la ley por sobre todas las cosas, porque en ello va la batalla por el respeto y la autodeterminación.

\section{Referencias}

Abbott, A. (1992). What do cases do? Some Notes on activity in sociological analysis. En Ragin, C. C. \& Becker, H. S. What Is a Case? Exploring the Foundation of Social Inquiry (pp. 53-82). Cambridge: Cambridge University Press.

Acselrad H. (Org.). (2004). Conflitos ambientais no Brasil. Río de Janeiro: Relume-Dumara.

Acselrad, H., Campello do Amaral Mello, C. \& Das Neves Bezerra, G. (2009). O que é justiça ambiental. Río de Janeiro: Garamond Universitaria.

Agua y Saneamientos, S. A. (AySA). (2013). Informe al Usuario. Recuperado el 9 de octubre de 2017, de http://www.aysa.com.ar/Media/archivos/684/Informe_al_Usuario_2013.pdf

Agua y Saneamientos S. A. (Aysa). (2009). Estudio socioeconómico y ambiental en la Cuenca Matanza Riachuelo. Vols. I, II, III y IV. Buenos Aires: AysA.

Akrich, M., Barthe, Y. \& Rémy, C. (2010). Sur la piste environnementale. Menaces sanitaires et mobilisations profanes. París: Presses des Mines. 
Argenpress. Agencia de noticias. (2014). Recuperado el 22 de octubre de 2016, de http://www .argenpress.info/2014/09/la-muerte-silenciosa-de-los-ninos-en.html

Bagnuolo, M. A. (2012). Diagnóstico Socio-ambiental de Villa Lamadrid. Mimeo.

Bernstein, M. \& Jasper, M. (1988). Les tireurs d'alarme dans les conflits sur les risques technologuiques. Enre intérets particuliers et crédibilité. Politix. Revue des Sciences Sociales du Politique, (44), Quatrième trimestre, 109-134.

Blanchon, D., Moreau, S. \& Veyret, Y. (2009). Comprendre et construire la justice environnementale. Annales de Géographie, 1(665-666), 35-60.

Brown, P. (1992). Popular Epidemiology and Toxic Waste Contamination: Lay and Professional Ways of Knowing. Journal of Health and Social Behaviour, 33, 267-281.

Bullard, R. D. (1994). Dumping in Dixie: Race, Class and Environmental Quality. San Francisco/ Oxford: Westview Press.

Capek, S. (1993). The "Environmental Justice" Frame: A Conceptual Discussion and an Application. Social Problems, 40(1), 5-24. (Special Issue on Environmental Justice).

Capek S. \& Gilderbloom, J. I. (1992). Community Versus Commodity: Tenants and the American City. Albany, NY: State University of New York Press.

Cefaï, D. (2007). Pourquoi se mobilise-t-on? Les théories de l'action collective (p. 576). París: La Découverte (Bibliothèque Du Mauss).

Cravino, M. C. (2008). Los mil barrios informales en el amba. Los Polvorines: Paidós/UnGs.

Di Chiro, G. D. (1998). Nature as Community: The Convergence of Environment and Social Justice. En Faber, D. (Ed.). The Struggle for Environmental Democracy: Environmental Justice Movements in the United States. Nueva York: Guilford.

Faber, Daniel (2005). Building a Transnational Environmental Justice Movement: Obstacles and Opportunities in the Age of Globalization. En Bandy, J. \& Smith, J. (Eds.). Coalitions Across Borders: Negotiating Difference and Unity in Transnational Struggles Against Neoliberalism. Nueva York: Roman \& Littlefield.

Fernández Bouzo, S., Casamiquela, S., Aizcorbe, M. \& Gil, M. A. Atravesando el río: agua, contaminación y saneamiento ambiental en la cuenca Matanza-Riachuelo. Video. Recuperado el 9 de octubre de 2017, de https://geaiigg.wordpress.com/page/3/ 
Firpo Porto, M. (2012). Movements and the Network of Environmental Justice in Brazil. Environmental Justice, 5(2), 100-104.

Fraser, N. \& Honneth, A. (2006). ¿Redistribución o reconocimiento? Madrid: Morata.

Fuks, M. (1996). Do discurso ao recurso: uma análise da proteção judicial ao meio ambiente do Rio de Janeiro. En Ferreira, L. \& Viola, E. (Eds.). Incertezas de sustentabilidade na globalização. Campinas: Unicamp.

Gamson, W. (1988). Political Discourse and Collective Action. International Social Movements Research, (1), 219-244.

Gibbs, L. (1992). Love Canal, My Story. Albany, NY: State University of New York Press.

Guha, R. (1989). Radical American Environmentalism and Wilderness Preservation: A Third World Critique. Environmental Ethics, (11), 71-83.

Harvey, D. (1996). Justice, Nature, and the Geography of Difference. Oxford: Blackwell.

Hilgartner, S. \& Bosk, C. L. (1988). The Rise and Fall of Social Problems: a Public Arenas Model. American Journal of Sociology, 94(1), 53-78.

Holston, J. (2009). Insurgent Citizenship in an Era of Global Urban Peripheries. City \& Society, 21(2), 245-267.

Informe Foro Hidrico. Causa Riachuelo. El agua no es confort (2015). Informe de la Campaña realizada por Foro Hídrico y la Cátedra de Ingeniería Comunitaria de la UBA. Recuperado el 9 de octubre de 2017, de https://es.scribd.com/document/260050083/2015-Informe -Foro-Hidrico-Causa-Riachuelo

Instituto Nacional del Agua (INA). (2003). Estudio para el diagnóstico del ascenso de las aguas en el conurbano bonaerense y la Ciudad Autónoma de Buenos Aires. Buenos Aires: Convenio INA-ETTO.

Martínez Alier, J. (2005). El ecologismo de los pobres. Conflictos ambientales y lenguajes de valoración. Barcelona: Flacso/Icaria.

Merlinsky, G. (2016). Cartografias del conflicto ambiental en Argentina 2. Buenos Aires, Ciccus/Clacso. Recuperado el 9 de octubre de 2017, de http://www.clacso.org.ar/libreria -latinoamericana/libro_detalle.php?orden=\&id_libro=1177\&pageNum_rs_libros= 0\&totalRows_rs_libros=1121 
Merlinsky, G. (2014). Cartografias del Conflicto Ambiental en Argentina. Buenos Aires: ciccus/ CLACso. Recuperado el 9 de octubre de 2017, de http://biblioteca.clacso.edu.ar/clacso/se /20140228033437/Cartografias.pdf

Merlinsky, G. (2013). Politica, derechos y justicia ambiental. El conflicto del Riachuelo. Buenos Aires: Fondo de Cultura Económica.

Naguib Pellow, D. \& Brulle, R. (Eds.). (2005). Power, Justice and the Environment. A Critical Appraisal of the Environment Movement. Cambridge: MIT Press.

Paavola, J. (2007). Institutions and Environmental Governance: A Reconceptualization. Ecological Economics, 63, 93-103.

Polkinghorne, D. (1995). Narrative Configuration in Qualitative Analysis. International Journal Of Qualitative Studies In Education, 8(1), pp. 5-23.

Sismondo, S. (2010). An Introduction to Science and Technology Studies. (2a . ed.). Chichester: Wiley-Blackwell.

Schlosberg, D. (2012). Justicia Ambiental y climática: de la equidad al funcionamiento comunitario. Revista Ecología Politica, (41), 25-35.

Snow, D., Burke Rochford, E., Worden, S. \& Benford, R. (1986). Frame Alignment Processes, Micromobilization and Movement Participation. Americal Sociological Review, 51, 464-481.

Stake, R. (1995). The Art of Case Study Research. California: SAGE

Taylor, C. (1993). El multiculturalismo y la "politica del reconocimiento". México: Fondo de Cultura Económica.

Tobías, M. (2016). El acceso al agua en Buenos Aires durante la era post-neoliberal: ¿¿derecho humano o commodity? En Merlinsky, G. (Comp). Cartografias del conflicto ambiental en Argentina 2. Buenos Aires, Clacso/ciccus.

Torres, H. (1993). El mapa social de Buenos Aires (1940-1990). Serie Difusión 3. Buenos Aires: Secretaría de Investigación y Posgrado, Facultad de Arquitectura, Diseño y Urbanismo, Universidad de Buenos Aires.

Vainer, C. (2004). Águas para a vida, não para a morte. Notas para uma história do movimento de atingidos por barragens no Brasil. En Acselard, H. et al. (Comps.). Justiça ambiental e cidadania (pp. 185-215). Río de Janeiro: Relume Dumara.

Young, I. M. (1990). Justice and the Politics of Difference. Princeton, Princeton University 


\section{Entrevistas por G. Merlinsky}

Alfredo, Foro Hídrico. (2006).

A. A., integrante de Foro Hídrico. (2013).

A. L., integrante de Foro Hídrico. (2014).

Foro Hídrico. (2003). Entrevista colectiva.

Juan Walter, integrante de Foro Hídrico. (2004).

V. L., vecino de Lamadrid. (2006).

Víctor F., integrante de Foro Hídrico. (2006).

Recibido el 12 de enero de 2015.

Aceptado el 9 de marzo de 2017. 